\title{
Structural and Biomechanical Study of Clarinet Reeds Made from Arundo donax
}

\author{
Masahiro Kawasaki ${ }^{1}$, Tadashi Nobuchi ${ }^{2 *}$, Yuta Nakafusi ${ }^{3 \dagger}$, Masateru Nose ${ }^{4}$, Masateru Shibata ${ }^{1}$, and \\ Makoto Shiojiri, 3,5, : \\ 1. JEOL USA Inc., 11 Dearborn Road, Peabody, MA 01960, USA. \\ 2. Kyoto University, Kyoto 606-8501, Japan \\ * Present address: 1-68 Kunobe, Shiga, 520-2353, Japan \\ 3. School of Science and Engineering, University of Toyama, Toyama 930-8555, Japan \\ ${ }^{\dagger}$ Present address: Hokunetsu Co., Ltd., 115 Takaginishi, Toyama 930-0106, Japan \\ 4. Faculty of Art and Design, University of Toyama, Takaoka 933-8588, Japan \\ ${ }^{5 .}$ Kyoto Institute of Technology, Kyoto 606-8585, Japan \\ Present address: 1-297 Wakiyama, Kyoto 618-0091, Japan
}

The best reeds for a wind instrument such as clarinet, oboe, and bassoon are manufactured from a cane of Arundo donax (A. donax; giant reed) that grows only in a few areas in the Var in France where the Mediterranean climate is very mild. However, all the reeds still do not satisfy players with their musical performance. In a box of commercial reeds, there can be a large variation in quality and it is very common that the odds to get a usable reed are only a small percentage out of a box. Therefore, the relationship of structure and physical properties with musical performance of the reeds is still demanded to investigate, although there are many studies even on the acoustics of the clarinet [1-5]. In this study, by using various microscopy techniques, we observed the structures of two commercial clarinet reeds made out of $A$. donax, one (\#1) that exhibited good musical performance and the other (\#2) that is unusable. The local indentation hardness and Young's modulus of different tissues in these reeds are also measured. The structural and biomechanical differences between the two clarinet reeds are discussed and compared with a previous result of hichiriki reeds made from canes of Phragmites australis (P. australis; common reed) [6].

Specimens for light microscopy (LM) were prepared by cutting the sample reeds \#1 and \#2 (Fig. 1) to transverse sections about $30 \mu \mathrm{m}$ thick with a sliding microtome, followed by double staining with safranin and fast green FCF, similar to the experiment for canes of $P$. australis [6]. Specimens for scanning electron microscopy (SEM) were prepared by cutting almost the same places as used for LM. Any coating treatment to avoid charging effects was not made on the specimen surfaces. SEM observation was performed with a JSM-7800F Schottky field-emission (FE) SEM, a JSM-7100FT Schottky FE-SEM at $40 \mathrm{~Pa}$ in low vacuum mode and an ORION NS helium microscope. The hardness and Young's modulus of different parts on the cross-sectioned surfaces of the reeds \#1 and \#2 were also measured with a Vickers indentation load of $5 \mathrm{mN}$ using a nanoindentation system (Fischerscope, H100C-XYp) [6].

Figures $2 \mathrm{a}$ and $2 \mathrm{~b}$ show LM images of transverse sections of reeds \#1 and \#2, respectively. In both samples, the size of vascular bundle $(\mathrm{Vb})$ including vascular bundle sheath $(B s)$ increases gradually with the distance inward from epidermis $(E p)$. The $V b$ s in reed \#1 occupy the transverse cross-section surface as large as $10 \%$ in number density and more homogeneously distributed as compared with reed \#2. With increasing $\mathrm{Vb}$ size inward from $E p$, the number of the sclerenchymatous $(S c)$ cells constituting its $B s$ fiber ring reduces, particularly near xylems $(X)$. Some of $V b s$ in central cylinder $(C c)$ are not completely enclosed with the $S c$ cells but are contacted directly with parenchyma $(P a)$ cells so that their $B s$ rings look discontinuous. The number ratio of these discontinuous $B s$ rings is comparatively larger in 
reed \#2 than in reed \#1. The size of $P a$ cells in $C c$ is smaller in reed \#1 than in reed \#2. A. donax almost resembles each other among its species in plant anatomical characteristics, but is different not only in scale of outer shape but also size of cells from P. australis [6], as their English names of 'giant reed' and 'common reed', respectively, imply. This is a reason why A. donax cannot be used for hichiriki reeds. LM distinguished each cell of the reeds by staining with safranin and fast green FCT while SEM identified the hard $S c$ and $B s$ tissues with a bright flat plane, where the cells with a thick cell wall are touched firmly, and the soft $P a$ cells with their holes have a thin cell wall. SEM, in particular, He ion SEM revealed a fine structure of cell walls with multilayers. The $B s$ ring which envelopes soft tissue such as $P$ and $X$ in $V b$ runs along the longitudinal direction of the cane and forms a strong string like a tube. This $B s$ string connects with a net of thin cell-walls of $P a$ cells that also continue along the longitudinal direction of the cane. It is considered that the acoustic quality of a reed is mainly ascribed to the shape and configuration of $V b$ surrounded by $P a$ cells. The tissue of the $B s$ fiber rings has much higher indentation hardness $H_{\mathrm{IT}}$ and Young's modulus $E_{\mathrm{IT}}$ than $\mathrm{Pa}$ tissue in the inner $\mathrm{Cor}$ and $\mathrm{Vb}$ tissues. This indicates that $B S$ can work as a string for vibration. Reed \#1 with good musical performance is associated with a cane where $V b$ with continuous $B s$ fiber rings are homogeneously distributed with higher proportion among a softer network of small $\mathrm{Pa}$ cells.

[1] J Backus, J Acoust Soc Am 33 (1963) 305.

[2] SE Stewart, WJ Strong, J Acoust Soc Am 68 (1980) 109.

[3] AH Benade, SN Kouzoupis, J Acoust Soc Am 83 (1988) 292.

[4] A Hirschberg et al, J Phys 4 (1994) 559.

[5] P Kolesil et al, Ann Botany 81 (1998) 151.

[6] M Kawasaki et al, Micros Res Tech 78 (2015) 260; Microscopy Today in press.

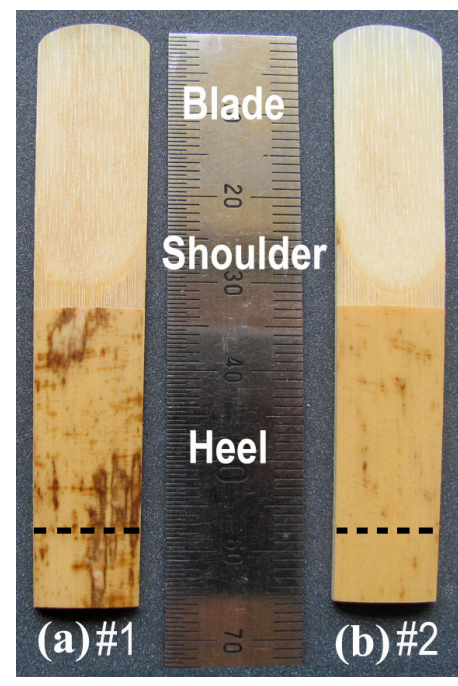

Fig. 1
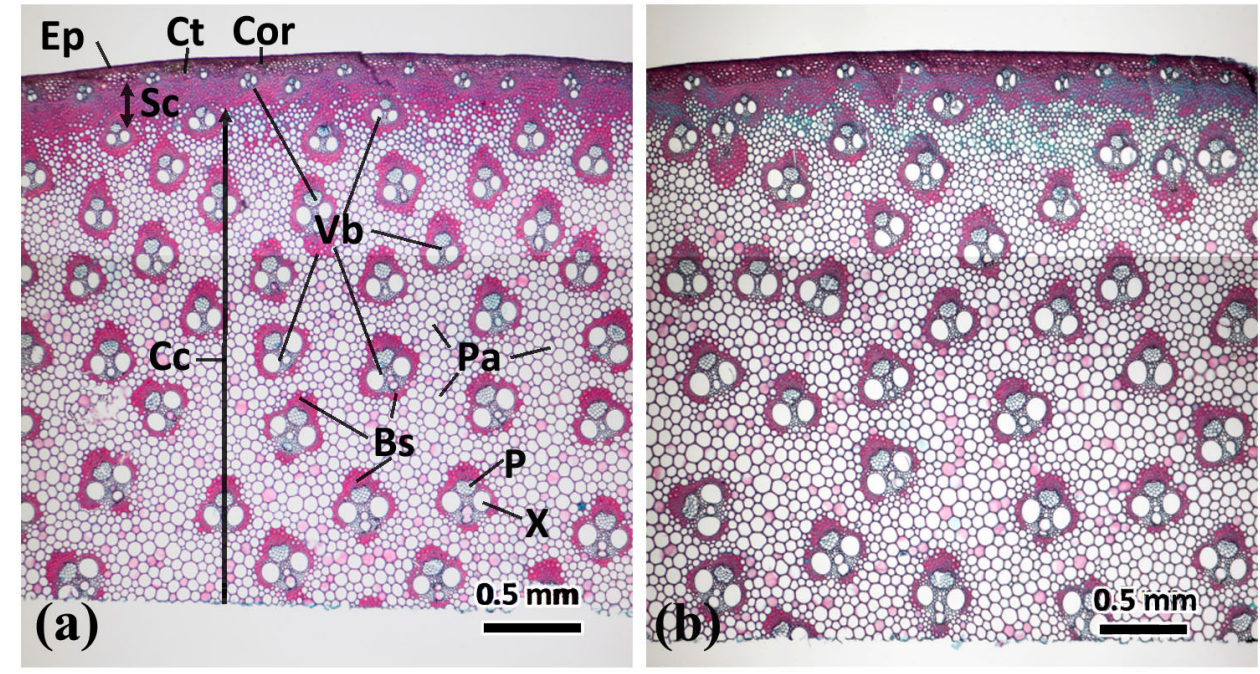

Fig. 2

Fig. 1. (a, b) Two commercial clarinet reeds, \#1 and \#2, respectively, manufactured from A. donax.

Fig. 2. (a, b) Light microscopic images of the thin transverse cross-sections of reeds \#1 and \#2, respectively. Ep: epidermis. Cor: cortex. $V b$ : vascular bundle. $B s$ : vascular bundle sheath. $C c$ : central cylinder. $P a$ : parenchyma cell. $C t$ : cells with thin cell wall. $S c$ : sclerenchymatous cell. $P$ : phloem. $X$ : xylem. For both (a) and (b), the top is the outmost surface of $E p$ and the bottom is the back surface of the reed that is not the face of pith cavity but a polished plane in $C c$. 